\title{
The Genetic Characterization of Chronic Pain Patients and Its Impact on Treatment Outcomes
}

\author{
RM Singa MD, MHS; LM Chargualaf MD; DG Conyack DO; HR Pak DO; AJ Iskander MD \\ Department of Anesthesiology and Perioperative Medicine, Saint Barnabas Medical Center, Livingston, NJ
}

Recent literature has discussed how pharmacogenomics can be utilized in pain management. There is little in the literature, however, describing the classification of the genetic profiles of patients in pain practices and subsequently, if knowledge of those profiles have improved outcomes. A relatively simple test involving a buccal swab from a patient can identify patients that possess a genetic alteration that alters drug metabolism, potentially putting these individuals at risk for adverse drug reactions or therapeutic failure. We aimed to quantify the distribution of CYP2C19, CYP2C9, and CYP2D6 alleles, in a population of pain patients who underwent genetic testing, and to elaborate if those patients demonstrated a decrease in pain scores after identifying potential altered medication metabolism.

After obtaining approval from the Saint Barnabas Medical Center institutional review board, we obtained records of all patients from the ambulatory pain center that underwent genetic testing. We noted their demographic information as well as the characterization of their metabolism for the CYP2C19, CYP2C9, and CYP2D6 alleles; medications identified by genetic testing as having substantial effect changes due to altered metabolism; medications identified by genetic testing to have an interaction with patients' other medications; medications and dosages at each visit; when the genetic test was performed, and pain scores from every visit.

We identified 70 patients who underwent genetic testing with complete characterization of CYP2C19, CYP2C9, and CYP2D6 alleles and identification of patients' drugs with metabolism genetically identified to be impacted by interaction with the altered alleles and/or other drugs consumed. The average age was 54 years, with a patient population composed of $59 \%$ women and $41 \%$ men.

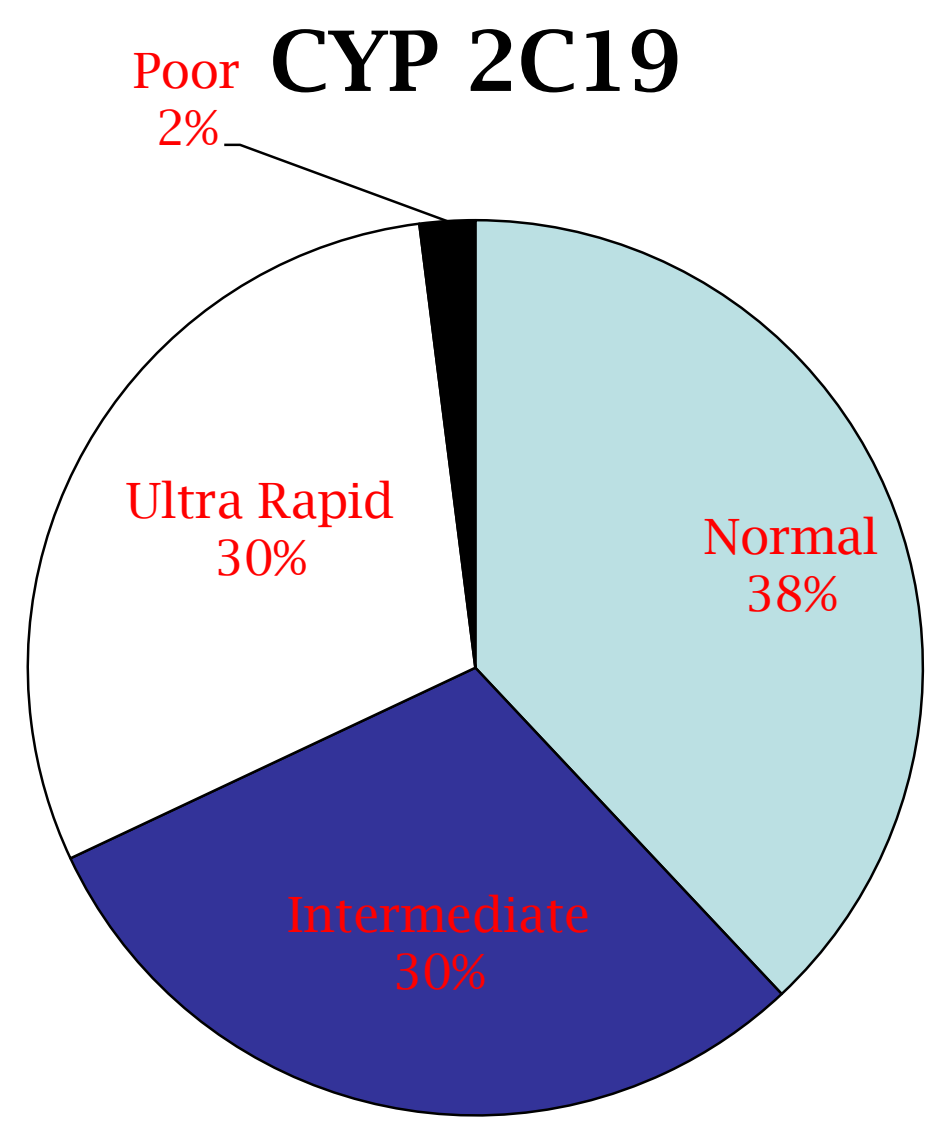

CYP 2C9

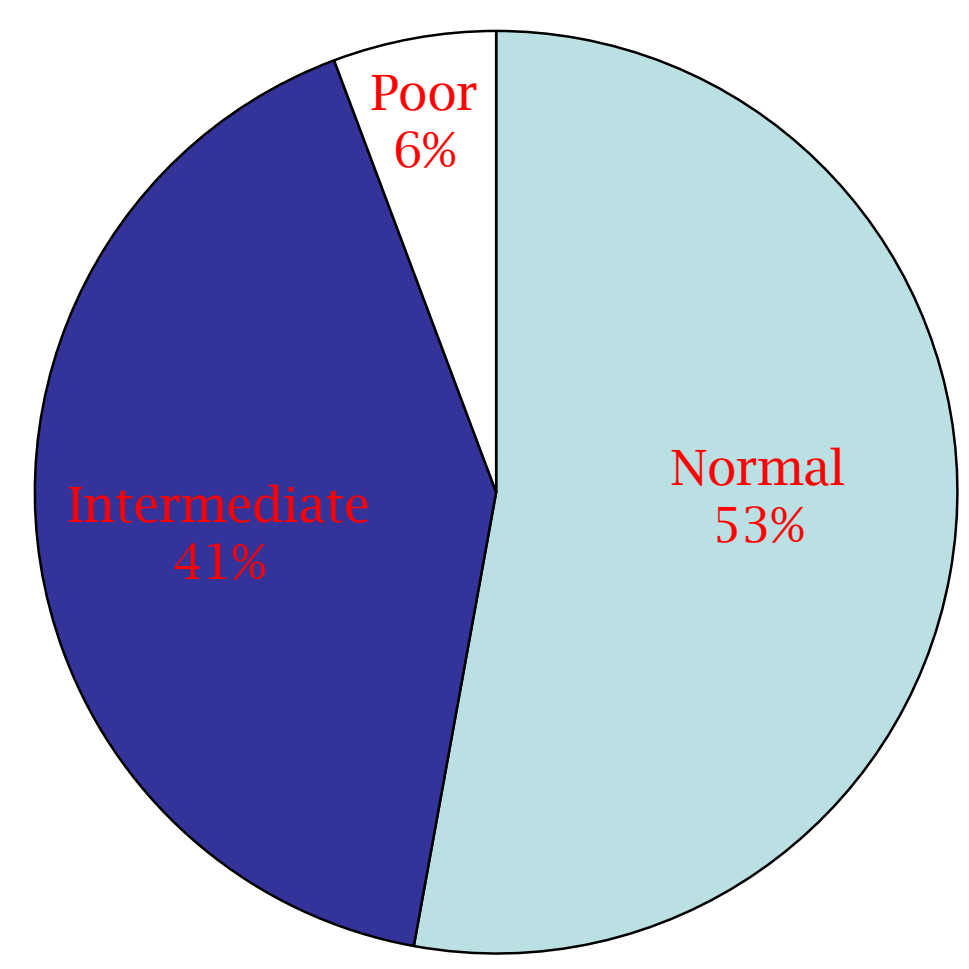

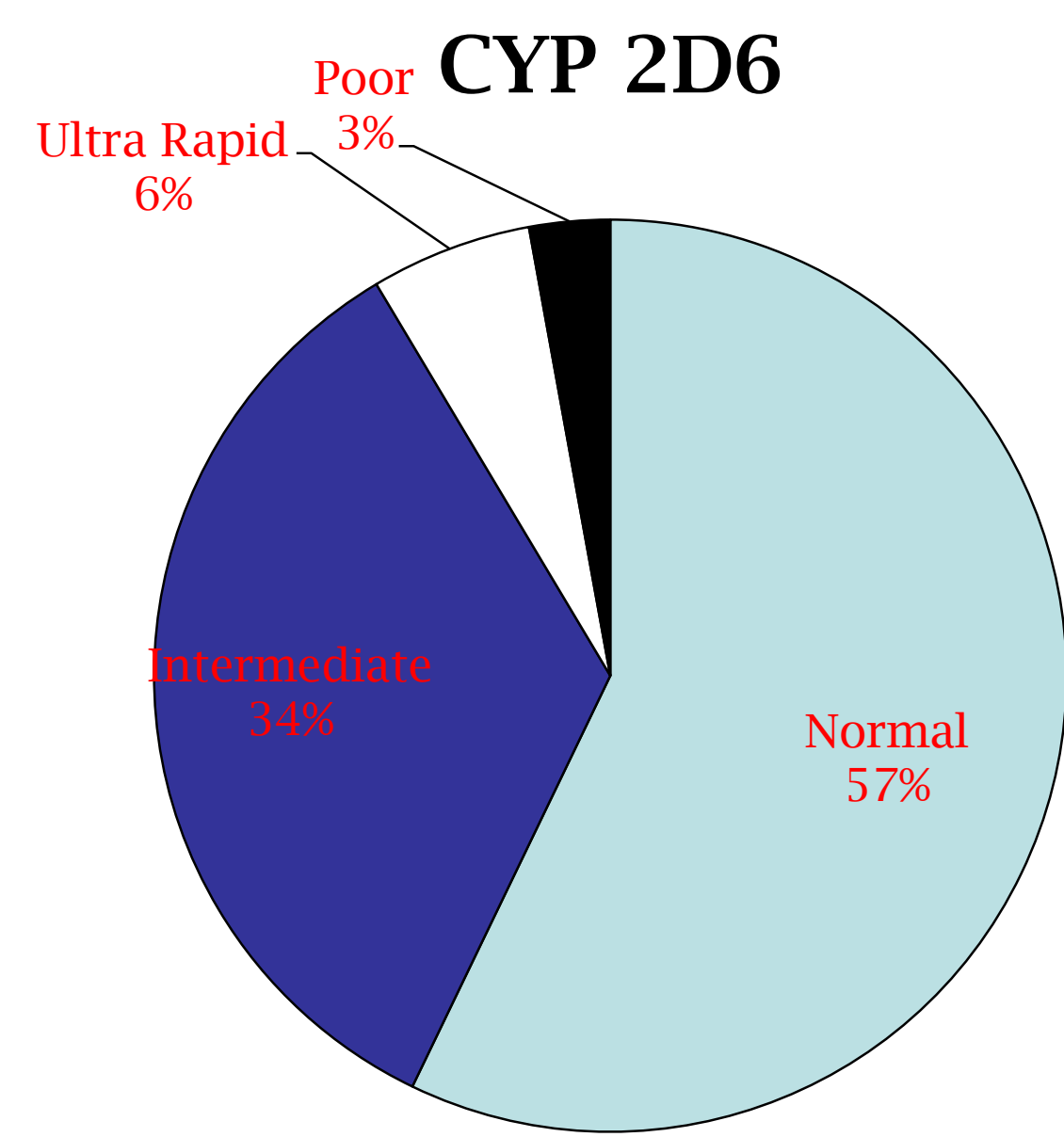

A total of 61 substantial known medication interactions existed amongst 25 patients, with $69 \%$ altered because of genetically identified altered metabolism with other medications and $31 \%$ due to altered allele activity. The average initial pain score on presentation was 8.6 points on a 10-point scale. The average greatest difference of pain scores on a 10-point scale after genetic testing was a decrease of 4.7 points from a patient's initial presentation to his lowest pain score and the average difference of pain scores on the same pain scale from a patient's initial presentation to his last visit identified at the clinic was a decrease of 2.8 points.

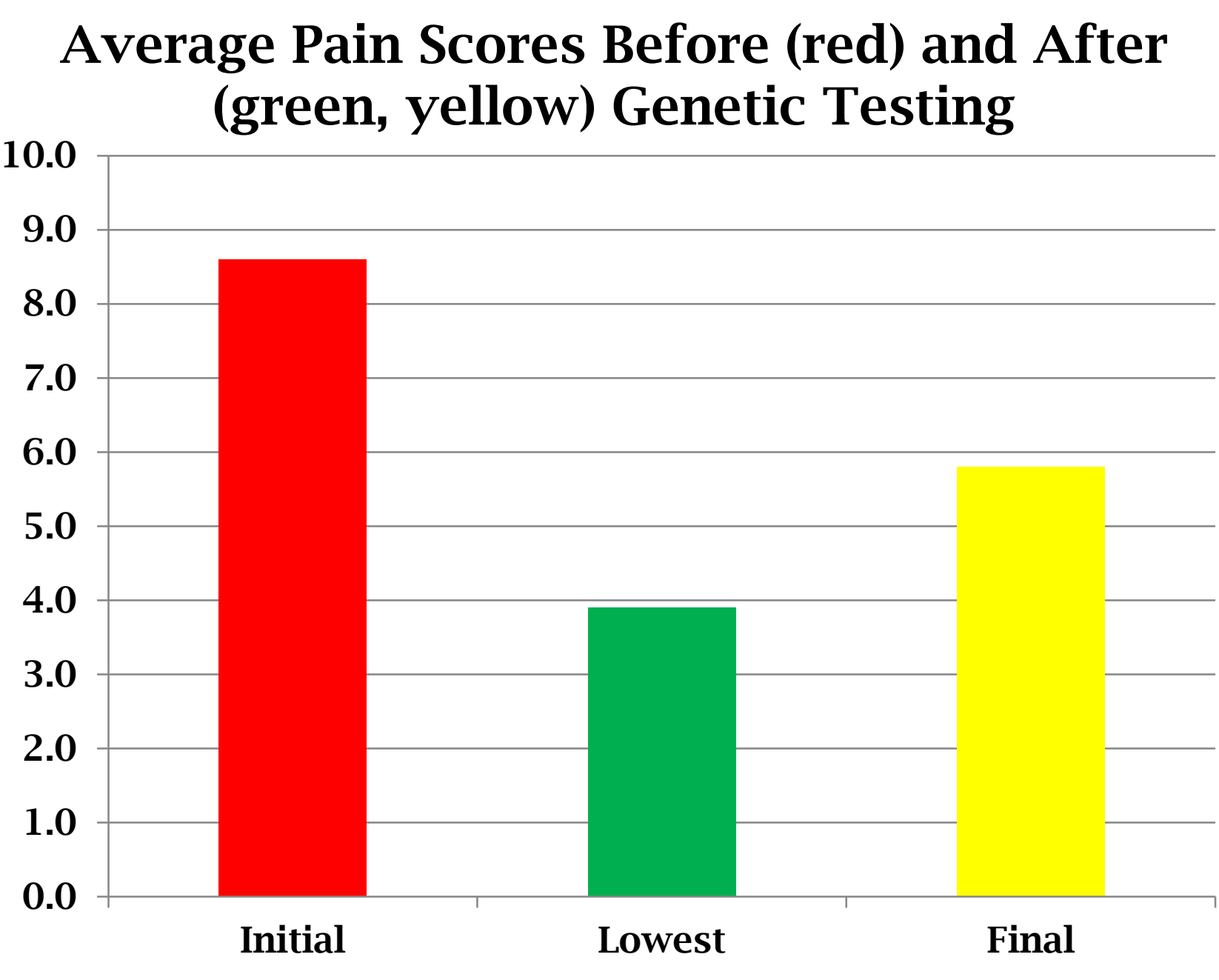

We found that the chronic pain population demonstrated a distinguishable difference in metabolic activity of the CYP alleles compared to the general population. In the CYP2D6 allele, there was a $70 \%$ decrease of poor metabolizers and $20 \%$ increase of extensive metabolizers. In the CYP2C9 allele, there was a $300 \%$ increase of poor metabolizers. In the $\mathrm{CYP} 2 \mathrm{C} 19$ allele, there was a $30 \%$ increase of ultra rapid metabolizers and a $50 \%$ decrease of poor metabolizers. Patients who underwent genetic testing had a reduction in pain scores after pain medications were changed in consideration of altered allele activity. A number of drug-drug interactions were identified genetically that had substantial impacts on its prescribed effect.

1. Branford R, Droney J, Ross JR. Opioid genetics: the key to personalized pain control? Clin Genet. 2012 Oct;82(4):301-10. 2. Gardiner SJ, Begg EJ. Pharmacogenetics, drug-metabolizing enzymes, and clinical practice. Pharm Rev. 2006 Sep;58(3):521-90. 